\title{
Geotechnical risk-informed decision-making in mining
}

\author{
JF Lupo Newmont USA Limited, USA
}

\begin{abstract}
Uncertainty and variability are ever-present in the fields of geomechanics and geotechnical engineering. These uncertainties and variabilities can introduce risk into mine facilities (open pits, underground, tailings storage facilities, etc.). Traditionally, the Factor of Safety (FS) has been used as a measure of anticipated performance. In other words, if the FS meets the acceptance criteria, it is anticipated that the facility will perform in an acceptable manner. However, the FS, by itself, does not communicate anything about risk, nor variability or uncertainty. Reliability methods allow calculation of a Probability of Failure (PF), which can be integrated into a risk assessment. However, a PF assumes that all of the critical elements of a design have been adequately addressed. In either case, the FS and PF, by themselves, are of limited utility if performance measures (deformation, piezometric head, etc.) are not also defined as part of the acceptance criteria.
\end{abstract}

Risk-informed decision-making (RIDM) provides an avenue to make informed risk decisions while providing a platform for defining the acceptance criteria. In this approach, there is a direct link between risk and performance. The RIDM process also allows the risk and acceptance criteria to be updated periodically, so that changes in the operation and/or performance can be considered. This approach is in contrast to the current practice where acceptance criteria are often defined without regard to the site conditions and where risk and acceptance criteria are treated as constants.

Keywords: risk-informed decision-making, geomechanics, acceptance criteria

\section{Introduction}

Risk and risk management are now quite common topics of discussion within the mining industry, and other industries as well. From a geomechanics and geotechnical engineering perspective, it is well-recognised that the failure of a pit slope, underground mine, tailings dam, etc. can carry significant safety and financial consequences. Therefore, the need to understand the likelihood of failure is crucial. Traditionally, mining facilities such as open pit slopes, underground mines, tailings storage facilities (TSFs) and waste rock facilities (WRFs), as well as other mine infrastructure, are designed, implemented and operated based on a set of prescriptive criteria (e.g. design criteria, construction technical specifications, and operational criteria). Prescriptive criteria provide an unambiguous set of numerical values or limits that must be achieved. Yet these criteria, by themselves, do not convey any information regarding risk to the overall operation.

In geomechanics and geotechnical engineering, a Factor of Safety (FS), is a commonly assigned as design criterion parameter. The FS is often referred to as a performance measure and, as such, is often taken as a de facto measurement of likelihood of failure. The assumption being that if a facility is designed to the desired FS, the likelihood of failure is quite low. Furthermore, it is often assumed the likelihood of failure is constant, and does not change over time. However, within the design, implementation and operational stages there are numerous opportunities where errors can be made or data misinterpreted, leading to a heightened risk profile.

Reliability methodologies, using statistical approaches to address uncertainties and variability, have been employed and allow the calculation of a Probability of Failure (PF) in addition to a FS. However, even these methods can be hampered by poor judgement and data limitations. Both PF and FS represent assumed outcomes, as neither can actually be measured. In addition, both PF and FS cannot be correlated to actual site measurements (e.g. how does a deformation measurement of $5 \mathrm{~cm}$ correlate to a FS or PF?), which may impact the assessment of risk. 
Finally, it must be recognised that risk is not a constant value; it changes and evolves over time due to changes at the mine operation and implementation (e.g. blast damage, construction methods, ground and surface water management, etc.). This constantly changing risk landscape requires a step-change on how the industry considers risk.

Risk-informed decision-making (RIDM) has been used in various industries since the 1960s to integrate risk into safety decisions (Federal Energy Regulatory Commission [FERC] 2016). RIDM is a continuous process in which decisions are made by evaluating if existing risks are tolerable and present risk mitigation measures are adequate, and if not, whether alternative risk mitigation measures are justified. The RIDM process acknowledges that human judgement and experience has a relevant role in decision-making, and that technical information cannot be the sole basis for decision-making. Notably, RIDM does not occur only at the design stage. Successful implementation of RIDM requires constant acquisition of performance data during implementation and operation to verify that performance meets expectations. The RIDM process provides a continuous 'report card' on the performance and health of the mine facilities. Within the geomechanics and geotechnical engineering realm, analyses are updated and evaluated based on performance measurements (stress, deformation, piezometric heads, etc.). This work, by itself, drives toward development of higher quality geotechnical and hydrogeological understanding of site conditions, which translates to better risk mitigation approaches.

\section{Factor of Safety/Probability of Failure}

Traditionally, the FS has been used as the predictive measure of performance and, by inference, risk (in the sense of likelihood of failure). Simply put, if the FS is within the desired acceptance criteria, the facility is anticipated to perform in an acceptable manner. However, while an acceptable FS value may be defined during the design stage, acceptable performance measures, such as deformation or piezometric head, are often not directly specified in the design work. Since an FS cannot be measured directly, it can only be considered an assumed outcome from the engineering analyses. In this sense, the FS represents a relative measure of the likelihood of failure. The term 'likelihood' being used here to signify this is a qualitative measure only, even though the FS is a numerical value.

One common criticism of FS is its inability to account for neither uncertainties nor natural variability (Duncan 2000; US Army Corps of Engineers [USACE] 2003). This has given rise to reliability methods, such as those presented by Lee and Lee (1998), Duncan (2000), Christian et al. (1994), Phoon (2004) and Zhang and Goh (2012), among others. Reliability methods employ statistical methods to 'bound' uncertainties and variability within the engineering analyses. The result of the reliability method is calculation of a PF, which can be used directly in risk assessments. However, as with the FS, the PF cannot be measured directly. Additionally, without defined performance measures linked to the design, it is not possible to assess whether the facility is responding as anticipated in the design. As a side note, reliability methods are integrated into what is commonly known as risk-based design (RBD), a subset of RIDM.

While reliability methods provide a more robust approach to assessment of risk, both PF and FS suffer from similar pitfalls, as discussed in the following section.

\subsection{Factor of Safety/Probability of Failure pitfalls}

By its very nature, geomechanics/geotechnical engineering requires human judgement and experience. From gathering data through engineering analyses, human judgement is required to interpret the data, conceptualise the site conditions, develop engineering properties of the materials, and define loading conditions. A discussion on the human element is presented later in this paper.

Field investigations, such as corehole drilling, geophysical surveys and trenching, are required to obtain information on the subsurface conditions at a site. The data derived from these investigations require interpretation in order to develop a conceptual model of the subsurface conditions. While this process may seem trivial, incorrect interpretation or inadequate site characterisation work can and often do result in poor understanding of the site conditions. Errors or poor understanding of site conditions can propagate as flaws 
in the subsequent design work, introducing an unknown and unquantifiable risk that cannot be reflected in an FS or PF calculation. The calculation of the FS or PF may be perfectly correct, but if the site conditions have been conceptualised incorrectly, these values become meaningless.

Samples (rock core and soil) obtained from the field investigations are subjected to laboratory testing to derive engineering properties that will be the basis for design work. Gill et al. (2005) provide some guidance on the number of test samples to be statistically significant. This, as with any guidance, assumes all of the critical lithologies (rock and soil) have been identified and sampled appropriately, which is predicated on executing a thorough field investigation program and development of a robust conceptual model. While there are standard sample preparation and testing procedures used to minimise testing errors, testing errors still do occur in sample preparation and testing. Within the industry, there is very little technical guidance or training on the interpretation of laboratory test data. Most often, the values provided by the testing laboratory are taken at face value and adopted for design, which has the potential to introduce errors in the design work.

As field and laboratory data are being collected, a site conceptual model is developed and continually evolving as more data is collected. This model describes the three-dimensional distribution of lithologies, structural features (faults, etc.), rock mass and soil characteristics, as well as the distribution of rock and soil engineering properties within the subsurface. The model also provides information on potential construction materials, if they are needed. The same is done to derive a hydrogeological conceptual model. The development of the geotechnical and hydrogeological conceptual models is one of the most important tasks in geomechanics/geotechnical engineering, yet very little attention is paid to proper model development and review. At this point, any errors in interpretations or assumptions from the field investigations and laboratory testing are 'baked' into the site geotechnical and hydrogeological conceptual models, which will ultimately add risk to the design.

Engineering analyses, based on the site geotechnical and hydrogeological conceptual models, are used to perform design, whether it is a pit slope, underground mine, TSF, etc. As part of these analyses, assumptions must also be made with regard to how the design will be implemented and operated for the life-of-mine. For example, a pit slope design may assume fully drained, depressurised slopes with nominal blast damage, an underground operation may assume a certain sequence (mining and backfilling) to achieve a desired stress distribution, a TSF dam may assume fully drained loading conditions in the foundation, or a waste rock facility may assume a certain loading rate to avoid development of undrained conditions in the foundation. Assumptions made on implementation and life-of-mine operation can often lead to conflict with actual site conditions. Assuming drained conditions for a facility, like a pit slope or TSF, is fine as long as drained conditions can actually be achieved in the time frame it is required. Making an assumption of nominal blast damage is okay if the operation has demonstrated experience with controlled blasting methods. If the assumptions made for implementation and operation are not valid or cannot be achieved, then the design is flawed, as are the FS/PF estimates.

\subsection{Acceptance criteria}

As presented earlier, an acceptance criterion (either for FS or PF, or both) is typically defined at the design stage, often before any geomechanics/geotechnical data has been collected. In most cases, the acceptance criterion is selected based on an individual's judgement or experience, dictated within an organisation's guideline/standard, or specified by a regulatory authority. In any case, the acceptance criterion is commonly specified without explicit consideration of the sites conditions, operation, and, more importantly, the risk profile of the facility. Additionally, acceptance criteria are typically treated as constants that do not change over time, even though there may be changes in the mine operation and implementation.

As noted previously, an acceptance criterion based solely on FS and/or PF is of little use if performance measures are not defined. Performance measures (e.g. deformation, piezometric head, stress/strain, etc.) must be part of the acceptance criterion so that there is direct linkage between design and operation. The acceptance criterion must be selected based on the following: 
- The risk profile, with a recognition that this may change over time as changes occur at the operation.

- The level of understanding of site conditions, which often changes over time as new data and observations are gathered from the site.

- The requirements for the specific application. For example, a long-term pit slope should have a different acceptance criterion than a short-term pit slope, yield pillars will carry a much different acceptance criterion than a development or crown/sill pillar, and a TSF located near a populated area would carry a different acceptance criterion than a TSF located in a remote area.

The development of an acceptance criterion for a facility should be part of the RIDM process, rather than a standalone process. By integrating the acceptance criterion into the RIDM process, risk decisions can now be considered on an ongoing basis (monthly, quarterly, yearly) rather than just once at the design stage.

\title{
3 Risk-based design/risk-informed decision-making
}

Zio and Pedroni (2012) define RBD as:

\begin{abstract}
"A decision-making process that provides a technically defensible basis for making decisions and helps to identify the greatest risks and prioritise efforts to minimise or eliminate them. It is based primarily on a narrow set of model-based risk metrics, and generally does not leave much space for interpretation. Considerations of cost, feasibility and stakeholder concerns are generally not a part of risk-based decision-making, which is typically conducted by technical experts, without public consultation or stakeholder involvement."
\end{abstract}

In contrast, RIDM is a process that uses a set of performance measures, together with other considerations, to inform decision-making. The RIDM process acknowledges that human judgement has a relevant role in decisions, and that technical information cannot be the unique basis for decision-making. This is because of inevitable gaps in the technical information, and also because decision-making is an intrinsically subjective, value-based task. In tackling complex decision-making problems involving multiple, competing objectives, the cumulative knowledge provided by experienced personnel is essential for integrating technical and nontechnical elements to produce dependable decisions.

Both RBD and RIDM processes have been widely used in other industries (aerospace, chemical, shipping, water storage dams, etc.) but are relatively new to the mining industry. Some general guidance (non-mining related) on the RBD and RIDM processes are provided by van Gelder et al. (2001), Olsson and Frantzich (2018), FERC (2016), Zio and Pedroni (2012), and others. In a general sense, RBD is embedded within the RIDM process, while RIDM provides further consideration to technical experience and judgement.

Figure 1 presents a graphic prepared by the author summarising the RIDM process for mining facilities. It is important to note that RIDM should be applied to the design, implementation, operation and closure for all mining facilities. The majority of available guidance on RIDM focuses primarily on design with some operational aspects (performance monitoring). Traditionally, RIDM has not been applied to implementation. This omission is particularly important as risks can be introduced during the implementation phase, (e.g. TSF construction, pit slope development, underground development, etc.).

As illustrated in Figure 1, a key component of the RIDM processes is a risk assessment. Risk assessments, whether qualitative or quantitative, provide the means for risk identification which feeds input into risk management and operational reassessment. It is at this point of the process that acceptance criteria, including performance monitoring measures, should be defined so that there is direct linkage between acceptance criterion and risk. Changes in risk and the risk profile should drive changes to the acceptance criterion. Otherwise, there is no clear way to judge the health of the facility (e.g. is it performing as anticipated so that risks are being mitigated?). 


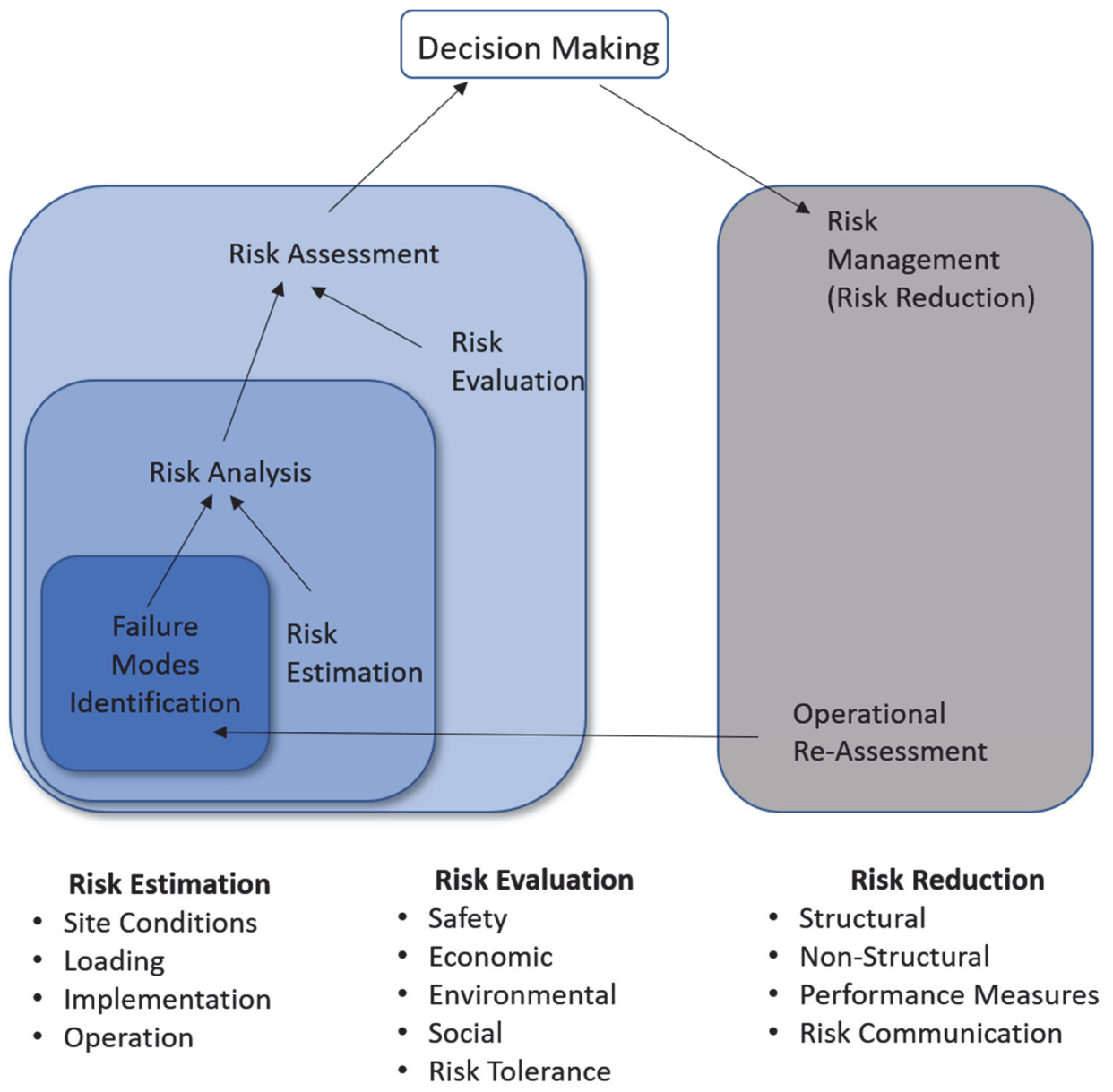

Figure 1 Risk-informed decision-making framework (after FERC 2016)

\subsection{The human element}

When assessing risks with respect to design, implementation, and operation, a qualitative measure of likelihood of failure is commonly adopted due to the presence of uncertainty and natural variability with geomaterials. Quantitative measures (such as PF) may also be used, assuming the data is statistically significant and representative. Uncertainty arises from our lack of perfect knowledge of a system, while variability reflects real differences in space and time of system variables (such as shear strength). As qualitative or quantitative measures of risk are employed, it is inevitable that the human element of judgement will be required (as highlighted in Section 2). The National Research Council (NRC 1994) suggests that uncertainty forces decision-makers to judge how likely risks will be underestimated or overestimated, whereas variability forces a decision-maker to cope with the certainty that differences exists. This leads us to conclude that the judgement (e.g. experience and bias) of the decision-maker(s) can have a significant influence when identifying and understanding risks related to mining facilities.

Siefert (2007) explains that humans are the sum of their experiences and education, as well as their emotional or non-rational reaction to circumstances. Researchers in human behaviour have shown that judgements and decisions of people are subject to numerous biases (Montibeller \& von Winterfeldt 2015), termed 'cognitive biases'. Cognitive biases are those that lead to judgements and decisions that violate commonly accepted normative processes. In other words, we may make judgements or decisions that do not fully take into account all of the information from a neutral point of view. Biases are present and shape our everyday lives. Some biases we are aware of, but most of the time we are unaware of our biases (Siefert 2007). With respect to risk, our cognitive biases influence our perception and tolerance to risk.

Well over one hundred cognitive biases have been identified (Figure 2). While it is not practical to understand all cognitive biases, it is important to recognise that biases shape our judgement and risk perception. What one 
person considers a high risk, another person may see as a low risk. In this context, it is easy to see how cognitive biases can shape the outcome of risk assessments and the RIDM process. If cognitive biases are not challenged or recognised, flaws can be introduced within the RIDM process itself, resulting in an underestimation or overestimation of risk and, potentially, the application of inappropriate risk mitigation measures.

Anytime judgement and experience are used in the risk process, recognise that biases may present that could dominate the process. Challenging the biases can be an effective tool for managing their influence, resulting in a more neutral or well-supported risk assessment.

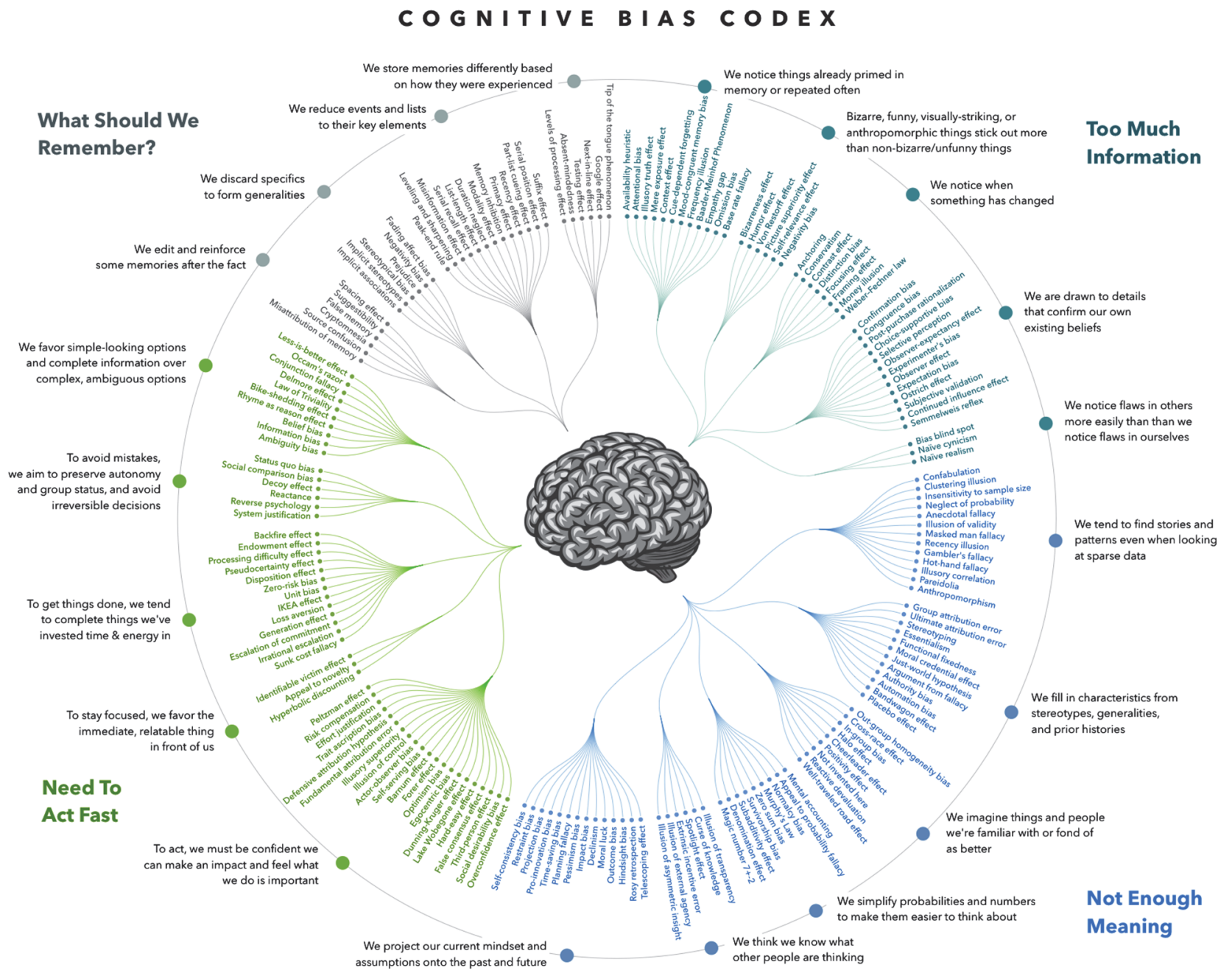

DESIGNHACKS.CO . CATEGORIZATION BY BUSTER BENSON - ALGORITHMIC DESIGN BY JOHN MANOOGIAN III (JM3) - DATA BY WIKIPEDIA

@creative
commons (1) (2) attribution·share-alike

Figure 2 Cognitive biases (Buster 2016)

\subsection{What does risk-informed decision-making look like?}

The application of RIDM basically follows the risk columns shown in Figure 1; namely risk estimation, risk evaluation, and risk reduction. Risk estimation focuses on potential failure modes, which will vary by the type of facility being considered (open pit, underground, TSFs, etc.). A number of formal processes, such as failure modes and effects analysis (FMEA), may be used to facilitate failure mode identification. These processes consider the potential failure modes for different aspects of the facility. For example, in an open pit slope the aspects may include:

- Bench-scale.

- Interramp-scale.

- Global-scale slopes. 
Within each aspect, the mode of failure (kinematic, rock mass, etc.) is considered. Other factors, such as rockfall, snow avalanche, landslide/debris flow, etc., that pose a risk are also considered. After each mode of failure is identified, the cause, effect and suggested corrective action(s) of the failure are documented. Each failure mode is also assigned a numerical ranking for likelihood of occurrence, likelihood of detection, and severity which culminates in a risk priority number (RPN). Note that the numerical ratings are subjective and prone to be influenced by cognitive biases.

While the FMEA and similar methods focus on the potential mode of failure, risk estimation must also consider the quality of the data, conceptual model, engineering design basis, design assumptions, performance measures (if applicable), etc. These can either be addressed separately or used as guidance for selecting numerical values for the RPN. Often these issues are overlooked. However, a proper evaluation of failure modes cannot be done without considering the quality of the data. The quality of data, particularly in geomechanics/geotechnical engineering, can be very subjective depending on judgement and experience. This data quality subjectivity can be addressed by having internal/external reviews.

Once the risk estimation is completed, the RIDM process moves to an assessment of risk with respect to:

- Safety.

- Economic.

- Environmental.

- Reputational.

- Social.

The focus of a risk assessment (RA) is to systematically look at each risk (as developed in the risk estimation step) to assess whether the risks are tolerable and proposed risk mitigation measures are adequate. If the risks are not tolerable or the risk mitigation measures are inadequate, then alternative risk mitigation measures are proposed. There are many guides on conducting RAs. Both Australian National Committee on Large Dams (ANCOLD 2003) and FERC (2016), although primarily geared toward water dams, are excellent resources for development of RAs and the application of RIDM.

Outcomes of the RA are used to inform decision-making and the development of methods to mitigate risks, termed risk reduction (Figure 1). It is at this point that the acceptance criterion should be developed (FS/PF and performance measures). By defining the acceptance criterion at this point, they become linked directly to the RA and embedded within the RIDM process. Note that the acceptance criterion can also be conditional. In other words, the acceptance criteria would change depending on the type of applied risk mitigation measures. A good example of this would be buttressing a pit slope versus conducting a layback on the pit slope. These are quite different risk mitigation measures, and could carry different acceptance criteria.

Once a decision has been made, the RIDM process includes periodic operational reassessments, as shown in Figure 1. This step is very important as it allows the risk profile to be re-evaluated to address any changes that may have occurred during operation. As with most geomechanics/geotechnical engineering projects, our understanding of site conditions increases overtime as we gain experience with the material behaviour and loading conditions. The operational reassessments provide the avenue to incorporate changing conditions into risk decisions.

It is noted that in all aspects of the RIDM process, communication is a critical component. Since the RIDM process encompasses a broad range of stakeholders from various disciplines, the method(s) of communication need to be tailored to the specific audience. At a minimum, the RIDM outcomes should identify potential risks with a discussion on how the risk is being mitigated through design, implementation and/or operation. The RIDM communications must also provide commentary on the data uncertainty and variability. 
On a final point, the RIDM outcomes must specifically define the acceptance criteria and performance measures for the facility. The connection between the risk decision and the acceptance criteria must be made very clear at this point, so that the monitoring program becomes a meaningful measure related to risk.

\section{Conclusion}

In the field of geomechanics/geotechnical engineering, there is always some level of uncertainty and variability, which can introduce risk into our facilities. Traditional methods of assigning an acceptance criterion based on FS, by itself, is not sufficient to communicate risk. Reliability methods, which allow calculation of $\mathrm{PF}$, provide a measure of probability that may be integrated into a risk assessment. However, a PF assumes that all of the critical elements of a design have been adequately addressed. By themselves, the FS and PF are of limited utility if performance measures (deformation, piezometric head, etc.) are not also defined as part of the acceptance criteria.

Ultimately, the acceptance criterion needs to be linked to risk, rather than be defined, as is often the case, before any data has been gathered. The RIDM process provides an avenue to make informed risk decisions while being a platform for defining the acceptance criteria. In this approach, there is a direct link between risk and performance. The RIDM process also allows the risk and acceptance criteria to be updated periodically, so that changes in the operation and/or performance can be considered. This is in contrast to the current practice where risk and acceptance criteria are constants.

\section{References}

Australian National Committee on Large Dams 2003, Guidelines on Risk Assessments, Australian National Committee on Large Dams, Hobart.

Buster, J 2016, The Cognitive Bias Codex, Wikipedia, viewed 6 December 2018, https://upload.wikimedia.org/wikipedia/ commons/6/65/Cognitive_bias_codex_en.svg

Christian, JT, Ladd, CC \& Baecher, GB 1994, 'Reliability applied to slope stability analysis', Journal of Geotechnical Engineering, vol. 120 , no. 12, pp. 2180-2207.

Duncan, JM 2000, 'Factors of safety and reliability in geotechnical engineering', Journal of Geotechnical and Geoenvironmental Engineering, vol. 126, no. 4, pp. 307-316.

Federal Energy Regulatory Commission 2016, Risk Informed Decision Making, Federal Energy Commission, Washington DC, viewed 6 December 2018, https://www.ferc.gov/industries/hydropower/safety/guidelines/ridm.asp

Gill, DE, Corthésy, R \& Leite, MH 2005, 'Determining the minimal number of specimens for laboratory testing of rock properties', Engineering Geology, no. 78, vol. 1, pp. 29-51.

Lee, IM \& Lee, MJ 1998, 'Optimization of rock slopes using reliability concepts', International Journal of Rock Mechanics and Mining Sciences, vol. 35, no. $4 / 5$.

Montibeller, G \& von Winterfeldt, D 2015, 'Cognitive and motivational biases in decision and risk analysis', Risk Analysis, vol. 35, no. 7, pp. 1230-1251.

National Research Council 1994, Science and Judgment in Risk Assessment, National Academy Press, Washington DC.

Olsson, F \& Frantzich, H 2000, 'A simple risk-based design method', Proceedings of the 3rd International Conference On Performance-Based Codes and Fire Safety Design Methods, The Society of Fire Protection Engineers, Gaithersburg.

Phoon, KK 2004, Towards Reliability-Based Design for Geotechnical Engineering, special lecture for Korean Geotechnical Society, Seoul, 9 July 2004.

Siefert, WT 2007, Cognitive Biases in Risk Management, master theses, University of Missouri, Columbia, http://scholarsmine.mst.edu/masters_theses/4590

US Army Corps of Engineers 2003, Engineering and Design: Slope Stability, US Army Corps of Engineers, Washington DC.

van Gelder, P, Roos, A \& Vrijling, H 2001, Risk-based Design of Civil Structures: Communications on Hydraulic and Geotechnical Engineering, Report No. 2001-01, Delft University of Technology, Delft.

Zhang, WG \& Goh, ATC 2012, 'Reliability assessment on ultimate and serviceability limit states and determination of critical factor of safety for underground rock caves', Tunneling and Underground Space Technology, vol. 32, pp. 221-230.

Zio, E \& Pedroni, N 2012, Risk-Informed Decision-Making Processes: An Overview, Foundation for an Industrial Safety Culture, Foundation for an Industrial Safety Culture, Toulouse. 